\title{
«Un État, deux Constitutions » : Analyse des dispositions transitoires de la Constitution congolaise du 6 novembre 2015
}

\author{
Vivien Romain Manangou*
}

\begin{abstract}
Adopted in institutional pain and popular protest, the Congolese Constitution of 6 November 2015 raises some fundamental problems. Indeed, its transitional measure establishes a kind of constitutional dualism, maintaining the institutions created by the 2002 Constitution along with those of the new fundamental text. This article analyses the causes and consequences of such a constitutional cohabitation, highlights the motives of the constitutional muddle and points out the desire for preservation of political power in the light of constitutional change.

Il est assez traditionnel, en droit constitutionnel, d'opposer " l'instabilité constitutionnelle de la France (avec ses 14 Constitutions de 1791 à 1958) [au] maintien outre-Atlantique ». ${ }^{1}$ Or, au jeu de cette instabilité constitutionnelle, les États de l'Afrique francophone, sont bien plus forts que la France - si on prend en compte leur brève existence en tant qu'État. $^{2}$

Ainsi, dans ce sport de la frénésie constitutionnelle, la République du Congo tient une place centrale, puisque depuis la vague de démocratisation des années $1990,{ }^{3}$ pas moins de
\end{abstract}

* Vivien Romain MANANGOU, Docteur en droit, Chargé d'enseignement à la faculté de droit de La Rochelle, 45 rue François de Vaux de Foletier, 17024 La Rochelle cedex 1 (Vivien.manangou@univ-lr.fr). Membre du Centre des études juridiques, environnementales et politiques (CEJEP).

1 HALPERIN (J.-L), « Changer de Constitution et par la Constitution : L'exemple historique des États fédérés d'Amérique jusqu'à la Première Guerre mondiale », Jus politicum, n9 - 2013, p. 1.

2 Voir sur le sujet, DOSSO (K.), « Les pratiques constitutionnelles dans les pays d'Afrique noire francophones : cohérences et incohérences "), Revue française de droit constitutionnel 2/2012 (n 90), p. 57-85; DIALLO (I.), « Pour un examen minutieux de la question des révisions de la Constitution dans les États africains francophones », Revue électronique Afrilex, mars 2015. Pour le cas spécifique du Sénégal, voir B. KANTE « Le Sénégal, un exemple de continuité et d'instabilité constitutionnelle » in Revue Juridique, Politique et Economique du Maroc, Numéro spécial, n²2, 1989.

3 On consultera utilement sur le sujet, l'ouvrage de Fabien EBOUSSI BOULAGA, Les conférences nationales en Afrique noire - Une affaire à suivre, Karthala, 1993. V. aussi, BAYART (J.-F), « La problématique de la démocratie en Afrique noire. La Baule, et puis après? », CNRS-CERI, juin 1990. 
cinq textes fondamentaux ont été adoptés. ${ }^{4}$ L'illustration récente de cette instabilité étant la promulgation d'un nouveau texte fondamental le 6 novembre $2015 .{ }^{5}$

Parce que l'État « dispose du monopole de l'édiction des règles de droit et de l'emploi de la force publique $»,{ }^{6}$ qu'il est « un ordre de contrainte [et donc] il institue la contrainte ${ }^{7}{ }^{7}$ Ainsi, l'État, comme le notait Carl Schmitt à propos de Kelsen, n'est que « normes et procédures $»{ }^{8}$ De ce point de vue, il est indispensable que lesdites normes et procédures soient codifiées dans un texte d'une normativité hors norme. Dès lors, la Constitution apparait comme « un texte normatif fondé sur un pacte collectif et volontaire tendant à construire un ordre stable de l'État, à travers l'organisation des pouvoirs, la réglementation de la production normative, et l'affirmation de principes et valeurs partagées $»{ }^{9}$

«Un État, deux Constitutions », formulé ainsi, le titre de cette analyse peut soulever diverses problématiques: « un État peut-il avoir deux Constitutions? » ou bien encore, « l'idée démocratique permet-elle une coexistence entre deux constitutions dans un même État? », etc.

Toutefois, cette multiplicité de questions n'empêchera pas le juriste de déceler entre les lignes la question combien intéressante de la succession entre deux ordres juridiques. Nous savons en effet que la rupture entre deux constitutions n'est jamais totale, ce qui s'explique par « La volonté de ne pas ignorer les textes antérieurs (...), les phénomènes illustrant, dans un même pays, la continuité des régimes, il faut aussi ajouter la permanence de certains principes ou règles sur lesquels il n'est pas envisagé de revenir (suffrage universel, grands principes fondamentaux $» .{ }^{10}$ De la sorte, « entre chaque discontinuité constitutionnelle, se tissent les procédures institutionnelles de la vie de l'État ». ${ }^{11}$

4 Acte fondamental adopté lors de la Conférence nationale; Constitution du 15 mars 1992, Acte fondamental de 1997, Constitution du 20 janvier 2002 et Constitution du 25 octobre 2015;

5 Le 25 octobre 2015, la République du Congo s'est dotée d'une nouvelle Constitution, approuvée par 92,66\% des Congolais, pour un taux de participation estimé par le ministère de l'Intérieur à $72,44 \%$. Le 6 novembre 2015, la Cour constitutionnelle annonçait les résultats définitifs, traduisant l'adoption du projet de nouvelle Constitution soumis au vote le 25 octobre par 94,32\% des suffrages exprimés à l'issue d'un scrutin marqué par une participation de $71,16 \%$. Dans la foulée, le président congolais promulguait la nouvelle Constitution. On pourra utilement consulter la nouvelle Constitution congolaise sur le lien ci-après : http://cour-constitutionnelle.cg/docs/constitution 25_10_2015.pdf.

6 PACTET (P.), MELIN-SOUCRAMANIEN (F.), Droit constitutionnel, Sirey, 30 éd., 2011, p. 35-36.

$7 \operatorname{KELSEN}(\mathrm{H}$.$) , « Aperçu d'une théorie générale de l'État », RDP, 1926, p. 572$ et 576.

8 SCHMITT (C.), Théorie de la Constitution, PUF, « Léviathan », 1993, p. 263.

9 PERLO (N.), « Les Constitutions provisoires, une catégorie normative au cœur des transitions constitutionnelles ", $A F D C$, Congrès de Lyon, 2014, p. 1.

10 VERPAUX (M.), Droit constitutionnel français, 2e éd, PUF, 2013, pp. 73-77.

11 SCHMITZ (J.), Essaie sur la théorie de l'institution du doyen Hauriou, Thèse, Université de Toulouse I Capitole, 2009, p. 333. 
Afin d'éviter tout malentendu, il convient de préciser que le concept de « double Constitution » n'est pas ici appréhendé dans le même sens que Carl Schmitt, ${ }^{12}$ pour qui « il n'existe pas seulement une constitution, la Constitution de droit positif; il soutient au contraire que l'on trouve derrière cette première Constitution une autre Constitution plus puissante, une deuxième Constitution foncièrement différente de la Constitution positive quant à son caractère et, pour ainsi dire, son essence et existence juridique $» .^{13}$ 《 Le concept de double Constitution », dans cette analyse, évoque les dispositions transitoires, contenues dans une Constitution adoptée qui permet à certaines dispositions de la Constitution abrogée de continuer à produire des effets. Par voie de conséquence, pendant une période limitée, deux Constitutions continuent de produire des effets dans un même ordre juridique.

En matière de changement de Constitution, João Paulo Pessoa prévenait que le « Pouvoir constituant originaire, qui pense une Constitution nouvelle qui révoque totalement la Constitution antérieure, sans aucun souci envers les situations déjà établies dans le régime constitutionnel précédent (...), atteindra difficilement le but de consolider le régime démocratique $»{ }^{14}$ Cela signifie que l'énoncé d'une disposition transitoire dans la Constitution à venir, permettant l'abrogation de la Constitution en vigueur, ne permet pas de lever toutes les ambiguités juridiques qui pourront naître d'une telle situation. En clair, il ne suffit pas de dire « la Constitution de 2002 est morte, vive la Constitution de 2015 », pour régler les conséquences juridiques d'une telle opération.

En effet, parce que la Constitution permet la manifestation « de l'activité dominatrice de l'État $»,{ }^{15}$ comme le soulignait Carré de Malberg, que l'on ne peut tolérer un vide entre deux ordres juridiques. Ainsi, « les dispositions transitoires figurent dans une loi ou un règlement nouveau et déterminent les modalités de son action dans le temps. Temporaires, elles présentent également un caractère secondaire, leur objet les vouant à accompagner une autre norme, la "disposition définitive" ». ${ }^{16}$ C'est pourquoi, « la raison d'être des dispositions transitoires est d'assurer la continuité de l'État en ce que les ruptures sont possibles et même souhaitables, mais il importe d'éviter des vides qui créeraient un instant de non-droit ou de négation de droit. Elles s'appliquent, de manière provisoire, en attendant l'accomplissement de certaines circonstances $\gg .{ }^{17}$

12 Voir les thèses développées par C. Schmitt, Théorie de la constitution, trad. fr., P.U.F., Léviathan, 1993, p. 151 et s.

13 JESTAEDT (M.), « La double constitution - Une stratégie positiviste », Jus politicum, nº-2011, p. 3.

14 PESSOA (J. P), « La transition constitutionnelle et le rôle des dispositions constitutionnelles transitoires : le cas du Brésil ", 9e Congrès français de droit constitutionnel, 26-28 juin 2014 (2014-11-13).

15 CARRE DE MALBERG (R.), Contribution à la théorie générale de l'État, Sirey, t. I, 1922, p. 269.

16 EVEILlARD (G.), Les dispositions transitoires en droit public français, Thèse de doctorat de droit public, Rennes 1, soutenue le 30 novembre 2005.

17 BOSHAB (E.), «Les dispositions constitutionnelles transitoires relatives à la Cour constitutionnelle de la République Démocratique du Congo », Fédéralisme Régionalisme [En ligne], Numéro 1 - 
De manière générale, les États qui changent de Constitution optent pour une période transitoire, régie par une Constitution provisoire ou un acte fondamental. Dans ce sens, $\mathrm{Ni}$ coletta Perlo reconnait trois objets aux Constitutions provisoires qui se concrétisent par trois décisions : " premièrement, la décision dé-constituante, deuxièmement, la décision constituante, et troisièmement, la décision constitutive d'un ordre juridique transitoire $»{ }^{18}$ Toutefois, cette catégorisation n'est véritablement valable que lorsque le changement résulte d'une rupture avec l'ordre politique précèdent, par une révolution ${ }^{19}$ ou encore par une situation exceptionnelle qui décrète la fin du régime précèdent, par exemple après la fin d'une guerre (France 1945) ou encore à la suite d'un consensus politique débouchant sur une volonté de rupture totale avec l'ordre ancien. ${ }^{20}$

Toutefois, dès lors qu'il n'y a pas une période de transition formalisée, comme c'est le cas au Congo, il n'existe pas de Constitution provisoire. Il revient alors au nouveau texte constitutionnel, fruit de la volonté politique des gouvernants, d'organiser la succession entre les deux ordres - on parle ainsi des dispositions transitoires.

On retrouve de plus en plus dans les Constitutions « les dispositions transitoires, qui ne s'appliquent que pour une durée déterminée (...) la doctrine a ainsi mis en évidence l'existence d'un "droit constitutionnel transitoire" qui permet le passage entre l'ordre juridique révolu et celui qui a vocation à être mis en place de manière définitive ». ${ }^{21}$ Cette idée suggère l'existence d'une « Constitution dans la constitution » et non pas une " petite Constitution », 22 que Marcel Prélot définissait comme « des normes, provisoires, parfois même formalisées, souvent uniquement matérielles, intermédiaires entre la Constitution révolue et la Constitution future encore au stade de projet $» .{ }^{23}$ 《Les dispositions transitoires figurent dans une [constitution], une loi ou un règlement nouveau et déterminent les modalités de

Premiers scrutins et contrôle de constitutionnalité en RDC : la mise en œuvre d'une constitution "régionaliste", Volume $7: 2007$, URL : http://popups.ulg.ac.be/1374-3864/index.php?id=561.

18 PERLO (N.), « Les constitutions provisoires, une catégorie normative au cœur des transitions constitutionnelles », précité, p. 9.

19 Ibid., pp. 19-24. Voir notamment la distinction très intéressante entre la Constitution provisoire de rupture (Libye) et la constitution provisoire de continuité (Tunisie) faite par N. Perlo.

20 C'était le cas de nombreux États africains avec les Conférences nationales des années 1990. Pour une analyse récente sur le sujet, voir Les Conférences nationales souveraines, Institut numérique, Rapport, 2013, en ligne : http://www.institut-numerique.org/31-les-conferences-nationales-souvera ines-522462d99e2a2.

21 GUILLOUD (L.), « Les révisions constitutionnelles induites par l'intégration européenne : l'introduction du désordre normatif dans la Constitution de 1958 », Association française de droit Constitutionnel, Congrès de Paris, 2008.

22 Voir sur le sujet, CARTIER (E.), « Les petites Constitutions : contribution à l'analyse du droit constitutionnel transitoire », Revue française de droit constitutionnel 2007/3 (n 71), p. 513-534.

23 PRELOT (M.), Précis de droit constitutionnel, Paris, Dalloz, 1949, pp. 307 et s. 
son action dans le temps. Temporaires, elles présentent également un caractère secondaire, leur objet les vouant à accompagner une autre norme, la "disposition définitive" ». ${ }^{24}$

La Constitution congolaise du 6 novembre 2015 consacre un titre entier aux dispositions transitoires. ${ }^{25} \mathrm{Du}$ fait de la cristallisation du débat politique autour de l'élection présidentielle et donc de la possibilité d'une nouvelle candidature du chef de l'État congolais, c'est essentiellement les dispositions sur l'exécutif qui étaient scrutées. Alors que, comme le notait justement le professeur Boshab, «Si elle ne [sont] pas judicieusement intégrées, [elles peuvent être] de simples leurres pour maquiller soit la mauvaise foi des gouvernants, soit leur incapacité de mettre en œuvre les innovations impulsées par le constituant ». ${ }^{26}$

Finalement, l'intérêt de ce sujet est de pointer l'importance des dispositions transitoires d'une manière générale, et particulièrement celles de la nouvelle Constitution congolaise, afin de convaincre qu'elles permettent la structuration définitive du nouvel ordre juridique (I), tout en étant un miroir des intentions politiques des tenants du changement du système (II).

\section{I) Une fonction organique : l'organisation de la phase transitionnelle entre deux textes fondamentaux}

«Le changement de la Constitution et par la Constitution $»^{27}$ prend tout son sens, en présence d'une abrogation de la Constitution par une nouvelle Constitution (A), tout en conduisant à un dualisme constitutionnel inédit (B).

\section{A. L'abrogation implicite de la Constitution du 20 janvier 2002}

Dans son Vocabulaire juridique, le doyen Cornu affirme que l'abrogation est la « Suppression, par une nouvelle disposition, d'une règle (...) qui cesse ainsi d'être applicable pour l'avenir ». ${ }^{28}$ Bien que le doyen Cornu, dans cette définition, ne fasse allusion qu'à « la règle ", nous considérons que, par extension, elle peut tout aussi s'appliquer à la norme constitutionnelle, qui est un ensemble de règles. De la sorte, le terme « abroger » marque l'idée d'une substitution définitive d'une norme constitutionnelle par une autre.

Cependant, il faut tenir compte du fait que l'abrogation de la Constitution se fait souvent de manière négative, c'est-à-dire que l'adoption d'une nouvelle Constitution implique

24 EVEILlARD (G.), Les dispositions transitoires en droit public français, Thèse, Université de Rennes 1 , soutenue le 30 novembre 2005.

25 Il s'agit du titre XXI, qui contient les articles 243 à 246.

26 BOSHAB (E.), « Les dispositions constitutionnelles transitoires relatives à la Cour constitutionnelle de la République Démocratique du Congo », précité, p. 2.

27 D'après le titre de l'article de HALPERIN (J.-L), « Changer de constitution et par la constitution : L'exemple historique des États fédérés d'Amérique jusqu'à la Première Guerre mondiale ", précité.

28 CORNU (G.), Vocabulaire juridique, Paris, PUF, 8e éd., 2000, p. 7. 
nécessairement l'abrogation de l'ancien texte fondamental. Il est donc assez rare de trouver une disposition qui pose expressément l'abrogation du texte en vigueur, tout au plus, il est juste précisé que la nouvelle Constitution rentre en vigueur après son adoption.

Ainsi, l'article 246 de la Constitution congolaise de 2015 dispose que « La présente loi, qui entre en vigueur dès sa promulgation, sera exécutée comme Constitution de la République du Congo ». ${ }^{29}$ Cette disposition implique l'entrée en vigueur immédiate de la nouvelle Constitution après sa promulgation. De même, elle conduit aussi à l'abrogation automatique de la Constitution antérieure. Toutefois, parce qu'aucune disposition ne prévoit cette abrogation, qu'elle est qualifiée d'implicite. De cette manière, l'abrogation implicite d'une Constitution peut être définie comme la suppression automatique de la Constitution antérieure, après la promulgation d'une nouvelle Constitution. C'est donc au moyen d'une déduction qu'on arrive à conclure que l'adoption et la promulgation de la nouvelle Constitution conduisent à l'abrogation de l'ancienne.

Cependant, les choses ne sont pas si simples. Il ne faut pas, en effet, perdre de vue que nous sommes dans « un intervalle de crise, un interrègne constitutionnel $»,{ }^{30}$ c'est-à-dire une période dans laquelle, « en apparence, semble être dominée plus par des équilibres entre des forces politiques que par le droit $» .{ }^{31} \mathrm{C}$ 'est pourquoi, « La nouvelle Constitution est élaborée selon le vœu émis par les gouvernants qui se trouvent au pouvoir au moment de l'émergence du nouvel État ou du nouveau régime ». ${ }^{32}$ Ainsi, « Le processus juridique de l'élaboration des textes est alors choisi par eux, de façon en définitive libre $»{ }^{33}$ En effet, il n'existe pas de disposition juridique qui les oblige à opter pour une voie plutôt qu'une autre, des solutions au détriment d'autres, sauf admettre des règles qui seraient d'une autorité supérieure, provenant de la nature, ou encore de la métaphysique en considérant une sorte de Grundnorm. ${ }^{34}$

Par voie de conséquence, comme le soulignait Esmein, « (...) on arrive fatalement à un certain point où le respect de la loi a pour unique garantie la conscience des autorités chargées de l'appliquer ». ${ }^{35}$ Ce constat « Ésmeinien » est valable au Congo. Il faut en effet souligner, comme Léon Duguit jadis, que « l'étude du droit public isolée de l'histoire et des

29 Constitution de 2015-Titre XXI. Dispositions transitoires et finales, Article 246.

30 CARRE DE MALBERG (R.), Contribution à la théorie générale de l'État, op. cit., p. 497.

31 PERLO (N.), « Les constitutions provisoires, une catégorie normative au cœur des transitions constitutionnelles », précité, p. 3.

32 VERPEAUX (M.), Droit constitutionnel français, op.cit., pp. 73-77. .

33 Ibid., p. 74.

34 Il s'agit d'une invention de Kelsen qui place au sommet de la pyramide, une norme hypothétique fondamentale, dite Grundnorm (le Grund désignant le fondement). Cette norme, de nature logicotranscendantale - Kelsen est en effet fortement influencée par le néokantisme - , est considérée comme une supposition nécessaire de l'esprit juridique, qui assure la cohérence de l'ordre juridique. Il ne s'agit pas d'une norme réelle, comme la Constitution : celle-là même devrait s'appuyer sur la Grundnorm imaginaire, placée au sommet de la pyramide.

35 ESMEIN (A.), « Note de jurisprudence parlementaire », RDP, t. XXIII, 1901, p. 314. 
faits est vaine et artificielle ». ${ }^{36}$ Partant de cette affirmation, pour comprendre le processus qui a amené à l'abrogation implicite de la Constitution congolaise du 20 janvier 2002, il faut connaitre quel était le contexte qui prévalait lors de la prise de décision du changement de Constitution.

Le parlementaire anglais De Lolme affirmait à la fin du XVIII ${ }^{\mathrm{e}}$ siècle que « le Parlement peut tout faire, sauf changer une femme en homme et un homme en femme $»{ }^{37}$ Le chef de l'État congolais a sans doute fait sienne cette maxime en changeant la Constitution sans prendre en compte toutes les incertitudes institutionnelles, juridiques et politiques qui résulteraient d'une telle opération. Nous le savons, « le contexte d'un changement de Constitution est toujours révolutionnaire (...) si, en effet, on définit la Constitution comme une décision politique globale sur le genre et le principe directeur de l'organisation politique, sa disparition et son remplacement par une autre impliquent une remise en cause de ce genre et de ce principe $»^{38}$. La difficulté dans le cadre congolais résulte de ce que le changement de Constitution n'était pas le fruit d'une remise en cause globale du système, bien au contraire. La décision de changer de Constitution répondait à la volonté du pouvoir en place de contourner les verrous constitutionnels posés par les articles 57, 58 et 185 de la Constitution du 20 janvier 2002. Ce rappel n'est pas anodin puisqu'il permet de comprendre la précipitation avec laquelle le référendum constitutionnel a été organisé. En effet, parce qu'une présidentielle était prévue en août 2016, qu'il fallait rapidement changer de Constitution sans passer par la case transition.

Nous savons que le changement de Constitution n'a véritablement de sens que s'il conduit à un changement en profondeur du régime. Dans ce sens, Jorge Miranda notait que « L'adoption d'un nouveau système constitutionnel par une communauté politique est un évènement rare (...) en général, elle découle de situations marquées par une crise profonde $» .{ }^{39}$ À partir de là, seule une transition permettra de juguler les conséquences éventuelles qui résulteraient d'un tel évènement. Ainsi, en 1991, après la Conférence nationale souveraine, une transition d'une année avait permis de poser les bases du nouveau régime. ${ }^{40}$ De même, en 1997, à la fin des hostilités entre les forces loyalistes et les insurgés « Cobra », une transition flexible de cinq ans fut initiée. ${ }^{41}$ L'objectif d'une telle période est de

36 DUGUIT (L.), RDP, t. XII, 1899, p. 526.

37 DE LOLME, Constitution de l'Angleterre, Van Harrenvelt, 1774, p. 129.

38 ROUVILlOIS (F.), Droit constitutionnel, Champs université, $4^{\mathrm{e}}$ édition, 2015, p.124. Voir aussi dans le même sens, G. LIET-VEAUX, La continuité du droit interne. Essai d'une théorie juridique des révolutions, Paris, Sirey, 1943.

39 MIRANDA, (J.), Manual de Direito Constitucional, 6. ed., Tomo II. Constituição. Coimbra : Coimbra, 2007, p. 101. Traduction de Jôao Paulo Pessoa.

40 Une loi fondamentale fut adoptée pour régir la période transitoire avec un Premier ministre de transition et la mise ne place d'un Conseil supérieur de la république comme Parlement de transition. La Constitution du 15 mars 1992 fut rédigée et adoptée par le peuple et le président Lissouba fut élu à la magistrature suprême en août 1992.

41 La Transition dura de 1997 à 2002, avec l'instauration d'un régime présidentiel assez autoritaire, un Conseil national de transition faisait office de Parlement transitoire, une Charte fondamentale 
faciliter le passage d'un ordre politique à un autre. Dans une telle configuration, la fin de la transition ne coïncide pas avec l'entrée en vigueur de la nouvelle Constitution. Au contraire, c'est l'organisation des élections et l'intronisation des nouvelles institutions qui sifflent la fin de la période transitoire. Dès lors, le changement de Constitution n'est pas implicite ou déduit, mais elle est expresse et explicite.

Cette expérience congolaise confirme les propos de Luc Heuschling, lorsqu'il affirmait que : « le droit est une technique de régulation sociale sophistiquée qui n'est pas sans pièges ni artifices. Le droit peut, d'un côté, dire une chose et, de l'autre, son contraire. Il peut mettre en avant, au début d'un texte, tel « beau » principe, pour ensuite, dans d'autres dispositions, affaiblir, voire annuler l'impact de l'affirmation précédente $» .^{42}$ Le piège dans cet imbroglio constitutionnel congolais, en l'absence d'une période transitoire, est de conduire à une rupture entre deux systèmes institutionnels, l'antérieur - parce qu'abrogé, et le nouveau - pas encore véritablement effectif.

En effet, en droit constitutionnel, comme l'observait Sébastien Ferrari, « le passage de l'ancien au nouveau système juridique engendre un ensemble de rapports inter-normatifs, parfois conflictuels (...) il s'avère alors nécessaire de réaliser une transition constitutionnelle $\gg .{ }^{43}$

Pour éviter cette difficulté organisationnelle, les concepteurs de la Nouvelle République $^{44}$ ont accordé une place centrale aux dispositions transitoires. De la sorte, si elles facilitent la gestion du passage de la Constitution de 2002 à la Constitution de 2015; elles vont aussi permettre « une application judicieuse de la règle de continuité de l’État ». ${ }^{45}$ Dans ce sens, le professeur Boshab fait un recours audacieux à la botanique pour comparer la transition entre deux systèmes constitutionnels, il note ainsi que : « (...) la nouvelle législation est la face apparente d'une superposition des sédiments : la sève des plus anciens contribuant à la jouvence de ceux que l'on aperçoit $» .{ }^{46}$ Dans ce sens, $R$. Odent considère, que l'abrogation implicite, " comme l'intervention d'un texte qui, sans faire aucune allusion aux textes qu'il abroge, se borne à édicter une règle de droit qui se révèle inconciliable avec des dispositions en vigueur $\gg .{ }^{47}$ Néanmoins, l'abrogation implicite que d'aucuns qualifient

fut adoptée pour régir la période transitoire. Une nouvelle Constitution fut adoptée le 20 janvier 2002.

42 HEUSCHLING (L.), « In cauda venenum. À propos des dispositions abrogatoires du projet de refonte constitutionnelle », Université de Luxembourg, Orbi, juin 2013.

43 FERRARI (S.), « De l'art du trompe-l'œil : l'abrogation implicite de la loi par la Constitution au service d'un continuum constitutionnel », RFDC, n83, 2010, p. 497.

44 Il s'agit du slogan porté par les partisans du changement de Constitution.

45 BOSHAB (E.), « Les dispositions constitutionnelles transitoires relatives à la Cour constitutionnelle de la République Démocratique du Congo », précité, p. 3.

46 Ibid., p. 3.

47 ODENT (R.), Contentieux administratif, t. I, Paris, 2007, p. 333-334. 
de « procédé de mise à mort ${ }^{48}$ n'est pas celle que nous mettons en avant dans le cadre de cette analyse. Il ne s'agit pas, en effet, de souligner le travail indispensable du juge dans l'épurement de l'ordre ancien, mais de constater que « ces dispositions sont, par le seul effet de l'intervention du nouveau texte, abrogées dans toute la mesure où leur maintien en vigueur est incompatible avec ce nouveau texte $»{ }^{49}$

Parce que le passé n'a que peu d'intérêt pour les auteurs des Constitutions, ${ }^{50}$ que lorsqu'une nouvelle Constitution remplace la précédente, la succession prend souvent la forme d'une abrogation. ${ }^{51}$ L'abrogation va alors conduire à une cessation des effets de l'ancienne Constitution, c'est-à-dire celle de 2002, mais pour l'avenir seulement, puisque continueront les conséquences qu'il a pu engendrer dans le passé. Ceci confirme bien le principe selon lequel « une norme peut disparaitre à la suite de l'intervention d'une norme d'un niveau au moins égal à la hiérarchie des normes qui la modifie ou l'abroge et pourvu que l'auteur de la norme l'ait voulu explicitement ou implicitement $»{ }^{52}$ Ainsi donc, l'abrogation implicite de la Constitution congolaise de 2002 n'entrave pas les principes constitutionnels en matière de changement de Constitution.

Néanmoins, dès lors que l'on souhaite « réconcilier l'État et la société, les gouvernants et les gouvernés, par le recours au droit $»,{ }^{53}$ il n'est pas possible de rompre totalement avec l'ordre ancien. En effet, si l'action dans le temps du nouveau texte est orientée vers l'avenir, sont conservées les conséquences de l'application de l'ancienne Constitution. ${ }^{54}$ Cette situation est amplifiée, notamment dans le cas de la Constitution de 2015, puisque le constituant fait le choix assez curieux d'un continuum institutionnel, confirmant au passage les propos du doyen Gicquel, lorsqu'il affirmait qu' « une Constitution achève son existence, tel Laza$r e$, et le cycle se reproduit, afin de répondre au besoin de légitimation du pouvoir » ${ }^{55} \mathrm{Ce}$ besoin a sans doute conduit à une situation inédite : l'existence de deux Constitutions produisant des effets en même temps.

48 Il s'agit d'un « outil juridictionnel d'épurement du corpus juridique de l'ordre ancien », Voir, FERRARI (S.), « De l'art du trompe-l'œil : l'abrogation implicite de la loi par la Constitution au service d'un continuum constitutionnel », art. cit., p. 503.

49 ODENT (R.), Contentieux administratif, op. cit., p. 337.

50 Voir ARDANT (P.), « Le temps dans les constitutions écrites », in La République; Mélanges en l'honneur de P. Avril, Paris, Montchrestien, 2001, p. 503.

51 FERRARI (S.), « De l'art du trompe-l'œil : l'abrogation implicite de la loi par la Constitution au service d'un continuum constitutionnel », art. cit., p. 498.

52 Ibid., p. 507.

53 PIERRE-CAPS (S.), Droits constitutionnels étrangers, Paris, PUF, 2010, p. 9.

54 Pour le cas des actes administratifs, Voir, AUBY (J.-M), « L'abrogation des actes administratifs », $A J D A, 1967$, nº $^{\circ}$, p. 131.

55 GICQUEL (J) et (J.-E.), Droit constitutionnel et institutions politiques, Paris, LGDJ, $28^{\mathrm{e}}$ édition, 2014, p. 206. 


\section{B. Le dualisme constitutionnel}

En principe, il est connu qu'avec l'abrogation, deux textes fondamentaux ne peuvent produire les effets au même moment. De cette manière, " la règle ancienne disparait au moment où la règle nouvelle entre en vigueur, sans que les effets pour le passé de la première soient remis en cause par la seconde $»,{ }^{56}$ C'est pourquoi, Sébastien Ferrari notait que « d'un point de vue chronologique, la coexistence entre deux Constitutions [était] impossibles ». ${ }^{57}$ Pourtant, il est possible de constater avec V.C.-E. Sénac, " [qu'elle] n'est pas totalement étrangère aux problèmes posés par la transition constitutionnelle $» .{ }^{58}$ De cette manière, le cas congolais en est une parfaite illustration.

Sans l'ambition de Dénis Béranger qui réclamait, il n'y a pas si longtemps, l'écriture de la Constitution anglaise non écrite, ${ }^{59}$ le concept de dualisme constitutionnel n'a pas pour objectif de conceptualiser sur une Constitution cachée, outre celle de 2015, adoptée dans la douleur. Il s'agit simplement de démontrer en quoi les dispositions transitoires, en cherchant à assurer une forme de continuité constitutionnelle, mettent en place un régime de juxtaposition de deux normes fondamentales. Bien entendu, un tel constat ne changera point - sur le plan de la théorie du droit, - la nature de la Constitution. Il s'agit tout simplement d'enrichir la compréhension des juristes, sur la particularité du système mis en place.

En effet, alors que les Congolais, dans leur grande majorité, croient qu'ils n'ont qu'une seule Constitution, celle de 2015, et que l'ancienne Constitution, celle de 2002, a été abrogée. Cette croyance est, en partie, inexacte. En réalité, il y a, en partie, deux Constitutions : celle de 2015 et celle de 2002 qui, en vertu de la Constitution de 2015, et notamment de ses dispositions transitoires, va rester partiellement en vigueur. Pour s'en convaincre, il suffit de lire le nouveau texte, et l'on s'aperçoit qu'il met en place un système complexe de dualité constitutionnelle ou de dédoublement. ${ }^{60}$

En effet, parce que le droit s'apparente à un compromis conclu entre des individus, que le droit est lui-même influencé par une contradiction intrinsèque, d'où la nécessité d'une conciliation permanente entre différentes normes. Ainsi, le doyen Hauriou soulignait à maintes reprises la contradiction fondamentale de la condition humaine. ${ }^{61}$ Cette contradiction peut aisément se déduire des articles suivants de la Constitution congolaise de 2015 :

56 FERRARI (S.), « De l'art du trompe-l'œil : l'abrogation implicite de la loi par la Constitution au service d'un continuum constitutionnel », art. cit., p. 498.

57 Ibid.

58 SENAC (V. C.-E), « Le constat juridictionnel de l'abrogation implicite d'une loi par la Constitution », RDP, 2008, n4, p. 1081.

59 Voir BÉRANGER (D.), Écrire la Constitution non écrite, PUF, Collection Leviathan, 2008.

60 Nous empruntons le terme à HEUSCHLING (L.), « In cauda venenum. À propos des dispositions abrogatoires du projet de refonte constitutionnelle », précité, p. 7.

61 HAURIOU (M.), Précis de droit constitutionnel, $2^{\mathrm{e}}$ éd., 1929. V. aussi, La pensée du doyen Hauriou et son influence, 1969. 
Article 243.

"Les traités et accords internationaux, les lois, les ordonnances et les règlements actuellement en vigueur, lorsqu'ils ne sont pas contraires à la présente loi, demeurent applicables tant qu'ils ne sont pas expressément modifiés ou abrogés ».

Article 244.

"Les institutions issues de la Constitution du 20 janvier 2002 fonctionnent jusqu'à la mise en place des nouvelles institutions sans pouvoir dépasser, pour les institutions pourvues par voie élective, l'expiration de leur mandat ».

Article 246.

"La présente loi, qui entre en vigueur dès sa promulgation, sera exécutée comme Constitution de la République du Congo ».

Ces articles, en apparence anodins, sont en réalité à la source d'un véritable « confusionnisme constitutionnel ». En effet, ils sont contradictoires, non pas en la forme, mais dans l'objectif assigné par le constituant.

De cette manière, l'article 243 permet une continuité du régime, en consacrant la continuité normative entre les deux textes constitutionnels. Puisqu'il pose le fait que toutes les normes internes ou externes, dès lors qu'elles ne sont pas incompatibles avec le nouvel ordre constitutionnel, sont applicables.

Quant à l'article 244, sans doute le plus caractéristique de la survivance de la Constitution de 2002, il permet aux institutions, pourtant régies et issues de l'ancienne Constitution, de demeurer en place, jusqu'à l'expiration du mandat pour les institutions élues. Ces deux articles nous permettent donc de dire que la Constitution de 2002, à travers le maintien de la réglementation, et surtout par l'entremise des institutions, telles que la présidence de la République et surtout le Parlement, demeure, au moins en partie en vigueur.

Enfin, l'article 246 confirme que juste après sa promulgation, la Constitution de 2015 est exécutée comme la seule Constitution de la République du Congo.

Dans cette configuration, il faut donc noter que, dans certains cas, lorsqu'il s'agira des institutions, c'est la Constitution de 2002 qui s'appliquera - puisqu'elles y sont issues dans d'autres, c'est la Constitution de 2015. De ce point de vue, comme le notait le professeur Jorge Miranda, pendant une transition, « il y a toujours un dualisme, pendant que la nouvelle Constitution se prépare, l'antérieur subsiste encore, pendant un délai déterminé ${ }^{62}$ Il ne s'agit donc pas d'une catastrophe constitutionnelle, dans la mesure où ce dualisme est limité dans le temps. En effet, l'article 244 qui établit la dualité constitutionnelle, la limite dans le temps, en posant que les institutions issues de la Constitution de 2002 continuent de fonctionner, sans dépasser pour les institutions élues, le mandat prévu.

62 MiRAndA (J.), Manual de direito Constitucionnal, $6^{\mathrm{e}}$ éd., Tomo II. Constitutuiçao, Coimbra: Coimbra, 2007, p. 209. Traduction de Jôao Paulo Pessoa. 
Ainsi donc, le Parlement congolais dont le mandat expire en juillet 2017 ne pourra pas excéder cette date. La question ne se pose plus pour la présidence de la République, dont l'élection a été anticipée le 20 mars 2016, pour sans doute répondre à cette urgence institutionnelle.

Toutefois, si sur le plan institutionnel, issue de l'article 244, la durée de la dualité constitutionnelle est bien limitée, comme nous l'avons souligné, ce n'est pas le cas pour l'article 243, notamment pour ce qui est des ordonnances et des règlements. En effet, il ressort de cet article que les actes administratifs, qui tiennent toujours leur validité d'une norme supérieure, seront évalués à l'aune non pas de la nouvelle Constitution, mais à l'aune du texte de 2002. A contrario, les actes pris après l'entrée en vigueur de la Constitution de 2015 seront évalués sous l'empire de cette dernière.

Cette situation, en apparence sans importance, peut avoir des conséquences inextricables, notamment en matière de droits. À titre illustratif, l'ancien article 10 posait la possibilité pour tout citoyen de désobéir en présence d'une atteinte manifeste au droit de l'homme. ${ }^{63}$ Or, lors des manifestations d'octobre $2015,{ }^{64}$ les opposants justifiaient le recours à la désobéissance civile par l'article 10 de l'ancienne Constitution. Ainsi, certains opposants emprisonnés avaient organisé leur défense autour de cet article. Dans la Constitution de 2015, adoptée en novembre, après les faits, cette disposition n'existe plus - dès lors, comment les accusés pourront-ils se prévaloir du droit à la désobéissance? La confrontation entre les deux ordres juridiques étant évidente, on ne peut donc pas évoquer « la force abrogatoire » de $J$. Trémeau, ${ }^{65}$ puisque les normes incompatibles ne disparaissent pas automatiquement avec l'adoption de la nouvelle loi. Au contraire, la transition entre les deux Constitutions se réalisera « au coup par coup, au gré des manifestations de la volonté du législateur ». ${ }^{66}$ Le rôle du législateur sera de premier plan, puisque dans de nombreux cas, l'application des dispositions constitutionnelles est conditionnée par l'adoption d'un acte législatif.

Cependant, c'est le juge qui devra assurer le « processus constant et progressif d'adaptation de l'ordre ancien à l'ordre nouveau ». ${ }^{67}$ Toutefois, la question sera de savoir si le juge de droit commun pourra devenir le juge de la garantie du respect de la nouvelle Constitution. Or, nous savons tous que cette compétence est une exclusivité du juge constituti-

63 Art. 10 de la Constitution de 2002 « Tout citoyen, tout agent de l'État est délié du devoir d'obéissance lorsque l'ordre reçu constitue une atteinte manifeste au respect des droits humains et des libertés publiques. L'ordre d'un supérieur ou d'une quelconque autorité ne saurait, en aucun cas, être invoqué pour justifier ces pratiques ».

64 Des nombreuses manifestations, réprimées dans le sang avaient été organisées par l'opposition réunie au sein du bloc IDC-FROCAD pour contester le recours au référendum pour changer la Constitution.

65 TREMEAU (J.), « La caducité des lois incompatibles avec la Constitution », AIJC, 1990, p. 252.

66 FERRARI (S.), « De l'art du trompe-l'œil : l'abrogation implicite de la loi par la Constitution au service d'un continuum Constitutionnel », art. cit., p. 502.

67 Ibid., p. 503. 
onnel. ${ }^{68}$ Dans ce cadre, il sera difficile d'aboutir à une abrogation progressive des normes incompatibles à la nouvelle Constitution, tant que seul le juge constitutionnel sera compétent. En France, c'est le juge administratif qui s'est évertué à assurer une adaptation entre l'ordre issu de la Constitution de 1946 et celui de la Constitution de $1958 .{ }^{69}$

On le voit, tout le travail consistera à « la mise en adéquation de l'ancien droit hérité du passé avec la nouvelle Constitution, opération qui demande des efforts plus ou moins importants aux acteurs du système juridique en fonction de l'écart entre ces deux ensembles de normes juridiques $»{ }^{70}$

Au-delà, en partageant la définition de la transition constitutionnelle de Cristina Queiroz, qui pose la transition constitutionnelle comme le « remplacement d'une Constitution par une autre, par l'appel au pouvoir constituant originaire, sans passer par les procédures de la rupture révolutionnaire qui, par définition, ne comprennent pas des règles constitutionnelles $» .{ }^{71}$ Nous pouvons affirmer, comme Nicoletta Perlo, concernant la Libye, que « la réussite d'un processus constituant est possible seulement à la condition que des règles informelles productrices d'un équilibre aient déjà été élaborées par les acteurs en présence $»{ }^{72}$ La difficulté avec le cas congolais est l'absence d'une phase transitoire et constituante, encline à formaliser le nouveau régime.

La précipitation du processus constituant a conduit à la violation des principes constitutionnels, confirmant les propos de G. Liet-Vaux, lorsqu'il affirmait que: « ce ne sont pas les actes de violence qui caractérisent les révolutions, mais plutôt les violations des dispositions relatives à la révision de la Constitution en vigueur $\gg .^{73}$

L'ordre constitutionnel ainsi créé tarde à se mettre en place, tant de nombreuses incertitudes institutionelles demeurent d'actualité.

\section{II) Un objectif politique : remettre le chef de l'État sortant au cœur du jeu institutionnel}

S'il est indéniable que les dispositions transitoires de la Constitution de 2015 ont pour objectif de faciliter la transition entre les deux ordres constitutionnels. Il n'en demeure pas moins que les « règles constitutionnelles sont des contraintes qui s'imposent aux hommes

68 Art. 72 al 2 de la Constitution de 2015: « Elle est juge de la constitutionnalité des lois, des traités et accords internationaux $»$.

69 Voir par exemple, CARBAJO (J.), L'application dans le temps des décisions administratives exécutoires, Paris, LGDJ, Bibliothèque de droit public, t. 134, 1980, pp. 184 et s.

70 HEUSCHLING (L.), « In cauda venenum. À propos des dispositions abrogatoires du projet de refonte constitutionnelle », précité, p. 12.

71 QUEIROZ (C.), Direito constitucional, As instituições do Estado Democrático e Constitucional, Sao Paulo: RT; Coimbra, 2009, p. 150. Traduction Jôao Paulo Pessoa.

72 PERLO (N.), « Les constitutions provisoires, une catégorie normative au cœur des transitions constitutionnelles », AFDC, Congrès de Lyon, 2014, p. 23.

73 LIET-VAUX (G.), « La fraude à la Constitution, essai d'une analyse juridique des révolutions communautaires récentes: l'Italie, Allemagne, France », RDP, 1943, p. 117. 
politiques, mais ce sont aussi des ressources qu'ils essaient de plier ou d'adapter à leurs objectifs ». ${ }^{74}$ Dans ce sens, l'objectif implicite des dispositions transitoires de 2015 était de remettre en selle l'initiateur de la « Nouvelle République $»{ }^{75}$ Ce qui passait par le maintien des institutions issues de la Constitution du 20 janvier 2002 (A), tout en rendant inopérantes les dispositions constitutionnelles empêchant un troisième mandat $(\mathrm{B})$.

\section{A. Un continuum institutionnel contestable}

En constatant que l'Afrique noire était devenue un véritable laboratoire de changements constitutionnels, I. M. Fall ${ }^{76}$ ne croyait pas si bien dire, tant « la réception et la répudiation du constitutionnalisme occidental ${ }^{77}$ semblent la règle. De ce point de vue, il s'agira dans cette sous-partie d'analyser les conséquences d'un changement de Constitution sur le fonctionnement des institutions, ${ }^{78}$ depuis l'adoption de la Constitution de la République du Congo de 2015.

Dominique Rousseau soulignait, il y a quelque temps, que « le succès de la Constitution est le résultat d'une transaction, d'une relation qui s'établit entre ce que la règle de droit offre en matière de formalisation, de neutralisation, d'objectivation des questions politiques et ce que les acteurs cherchent à un moment donné pour atteindre leurs objectifs $»{ }^{79}$ En organisant un référendum en octobre 2015, le pouvoir congolais espérait changer de république, tout en maintenant les institutions et donc les hommes chargés de les administrer. Ce paradoxe a des conséquences évidentes sur le fonctionnement de l'État congolais après l'adoption de la nouvelle Constitution.

En règle générale, lorsqu'une nouvelle Constitution rentre en vigueur, elle crée forcément des nouvelles institutions. Il y a donc lieu de prévoir une disposition particulière dans la Constitution, qui organise la transition dans le temps. ${ }^{80}$ Ainsi, l'article 244 accorde un bail de re-légitimation aux institutions dont les membres sont élus: la présidence de la République et le Parlement, en posant qu'ils pouvaient continuer à fonctionner jusqu'à l'élection des nouvelles institutions.

Cet article est donc à la base de ce que nous qualifions de continuum institutionnel, c'est-à-dire une continuité des institutions issues d'une Constitution abrogée, après la

74 MENY (Y.), Le système politique français, Montchrestien, 1991.

75 Telle est la dénomination du nouveau régime issu de la Constitution de novembre de 2015.

76 FALL (I. M.), Évolution constitutionnelle du Sénégal, Dakar, CREDILA, 2007.

77 GICQUEL (J.) et (J.-E.), Droit constitutionnel et institutions politiques, op. cit, pp. 399 à 401.

78 Il est possible pour aller plus loin sur le sujet, concernant le Niger et le Benin, de lire la thèse de Mamadou GAZIBO intitulée, La problématique de la consolidation démocratique: les trajectoires comparées du Bénin et du Niger, Université de Montesquieu-Bordeaux IV, 1998.

79 ROUSSEAU (D.), « Les constitutionnalistes, les politistes et le "renouveau” de l'idée de constitution », in Droit et Politique, CURAPP, Paris, PUF, 1993, p. 40 et ss.

80 HEUSCHLING (L.), «In cauda venenum. À propos des dispositions abrogatoires du projet de refonte constitutionnelle », précité, p. 9. 
promulgation de la nouvelle Constitution. Le continuum institutionnel vise donc à favoriser le changement de régime, sans pour autant conduire au changement institutionnel immédiat. Il faut par ailleurs noter que concernant les institutions congolaises élues, notamment la présidence de la République, le mandat courait jusque août 2016. ${ }^{81}$ Quant au Parlement, des élections législatives devraient être organisées en juillet 2017. La question était donc de savoir comment on pouvait se prévaloir d'un nouveau régime, tout en conservant les institutions de l'ancien, sans donner l'impression que le changement de Constitution n'était pas simplement « un processus d'ajustement de la norme fondamentale? $»^{82}$

Concernant la présidence de la République, clé de voûte du régime dans la Constitution de 2002, ${ }^{83}$ il s'agissait de savoir comment elle pouvait s'adapter au changement de Constitution sans perdre sa légitimité. En effet, à partir du moment où le chef de l'État convoquait un référendum pour changer de Constitution, sans passer par une période transitoire, il était légitime de s'interroger sur la constitutionnalité du président en place. Ce doute s'explique par le fait que le président de la République tient sa légitimité de son élection - « la légitimité doit être prise dans le sens de la conscience du moment ${ }^{84}$-, mais son impérium, de la prestation du serment devant la Cour constitutionnelle. ${ }^{85}$ Ainsi donc, à partir du moment où la Constitution par laquelle le chef de l'État tire son impérium est abrogée, l'on peut sérieusement se poser la question de la validité de la fonction présidentielle. Cette question explique sans doute l'anticipation de l'élection présidentielle le 20 mars dernier. ${ }^{86}$ Dans ce sens, l'anticipation de l'élection présidentielle permettait à l'ex-futur président de la République de prêter serment devant la Cour constitutionnelle, et cette fois-ci sous l'empire de la Constitution de $2015 .{ }^{87}$ Auréolé par le suffrage universel, le chef de l'État, nouvellement élu, pouvait donc se prévaloir de la légitimité que lui conférait la nouvelle loi fondamentale. À vrai dire, le chef de l'État congolais avait fait sienne cette déclaration de F. Mitterrand concernant la France: « la politique [du Congo], je l'ai moi-même définie et [...] elle est conduite sous mon autorité. Je ne surprendrai personne [...] en ajoutant que le chef de

81 Ayant prêté serment le 16 août 2009, dans le cadre de son septennat, le mandat du président Sassou, en vertu de la Constitution de 2002, prenait fin le 15 août 2016.

82 ATANGANA AMOUGOU (J. L.), « La constitutionnalisation du droit en Afrique : l'exemple de la création du Conseil constitutionnel camerounais », AIJC, XIX, 2003, pp. 45-63.

83 Sous la Constitution du 20 janvier 2002, le président de la République était le centre névralgique du régime. Voir sur le sujet, notre thèse, intitulée, Le cas de la République du Congo. Un exemple de régime constitutionnel autoritaire, Université de La Rochelle, 2014, 543 pages.

84 MELEDJE (D. F.), « La révision des constitutions africaines dans les États africains francophones. Esquisse de bilan ", RDP, 1992, $\mathrm{n}^{\circ} 1$, pp. 111-113.

85 Article 69 al.2 de la Constitution de 2002 : « Le serment est reçu par la Cour constitutionnelle en présence de l'Assemblée nationale, du Sénat et de la Cour suprême ».

86 Le 20 mars 2016, lors du $1^{\text {er }}$ tour de l'élection présidentielle, Monsieur Sassou a été proclamé vainqueur par la Cour constitutionnelle avec un plus de $60 \%$ de suffrages.

87 L'alinéa 2 de l'article 76 de la Constitution de 2015 dispose que : « La prestation de serment du Président de la République élu intervient vingt (20) jours au plus tard après la proclamation des résultats définitifs de l'élection par la Cour constitutionnelle». 
l'État ne peut dépendre que de l'idée qu'il a de l'intérêt public et des suffrages du peuple qu'il l'a élu ». ${ }^{88}$

Cependant, s'il est possible d'accorder un brevet de satisfecit concernant la fonction présidentielle, en ce que l'anticipation de l'élection présidentielle a permis une re-légitimation. Néanmoins, il est possible de s'interroger sur les incertitudes qu'il résulte du reste de l'architecture institutionnelle et donc, in fine, du président de la République lui-même.

Nous savons que la fonction présidentielle au Congo est conçue pour « faire valoir la continuité au milieu des combinaisons ». ${ }^{89}$ C'est pourquoi, dans la Constitution de 2002 , comme dans celle de 2015, le président de la République est au carrefour des institutions. Pour parodier Michel Debré, nous dirons que la Présidence de la République est « une institution de sollicitation $» .^{90}$ De cette manière, cette sollicitation conduit à prendre une série de mesures ayant des incidences sur les rapports institutionnels. Ainsi, le chef de l'État, après l'adoption de la nouvelle loi fondamentale, entretient des relations assez ambiguës avec le Parlement.

En effet, la Constitution de 2002 ne prévoyait aucune forme de responsabilité entre le président de la République, chef du gouvernement, et la chambre délibérative. ${ }^{91}$ La seule responsabilité qui existait entre le gouvernement et l'Assemblée nationale était informative. Les députés n'ont donc pas reçu mandat pour recevoir la déclaration de politique générale du gouvernement et, éventuellement, censurer ce dernier, en cas de désaccord politique. Or, dans la Constitution de novembre 2015, le gouvernement est responsable devant le Parlement, qui peut engager sa responsabilité. ${ }^{92}$ De même, le président de la République peut dissoudre l'Assemblée nationale. ${ }^{93}$ Ces deux articles marquent une différence fondamentale entre les deux ordres constitutionnels, et mettent à mal le continuum institutionnel. Il y a en effet une opposition entre deux conceptions des rapports institutionnels. D'une part, une conception présidentialiste, avec une absence de responsabilité politique gouvernementale devant la chambre basse du Parlement ou, pour parler comme le doyen Gicquel, « une Constitution présidentialiste $» .{ }^{94} \mathrm{D}$ 'autre part, une conception plus atténuée du présidentialisme avec une prégnance parlementariste. À partir de là, la difficulté réside en ce qu'il est demandé à des parlementaires, élus sous un régime de l'impunité politique (absence du danger de dissolution), d'accepter les foudres présidentielles d'une dissolution. De l'autre côté,

88 Entretien à Libération, 10 mai 1984.

89 Nous empruntons cette formule au Général de Gaulle, Discours prononcé à Épinal, le 29 septembre 1946.

90 Michel DEBRE, Discours devant le Conseil d'État, 27 août 1958.

91 Dans ce sens, l'article 114 de la Constitution de 2002 disposait que : « le président de la République ne peut dissoudre l'Assemblée nationale. L'Assemblée nationale ne peut démettre le Président de la République ».

92 En effet, l'article 139 de la Constitution de 2015 prévoit que : «L'Assemblée nationale peut, par le vote d'une motion de censure, renverser le Gouvernement ».

93 Article 138 pose que : « Le Président de la République peut dissoudre l'Assemblée nationale ».

94 GICQUEL (J.) et (J.-E.), Droit constitutionnel et institutions politiques, op. cit, p. 499. 
dès lors que les parlementaires représentent le peuple, qu'ils ont été élus sans avoir reçu mandat de censurer le gouvernement, comment peuvent-ils se prévaloir de ce pouvoir à la suite d'un changement de Constitution?

À voir de plus près, l'actuel Parlement ne peut, comme l'y oblige la nouvelle Constitution, à travers son article $103,{ }^{95}$ recueillir le programme d'actions du gouvernement, car les députés n'ont jamais reçu mandat pour cela. Il faut dire, parce que l'élection est un processus de légitimation du pouvoir, ${ }^{96}$ que le mandat de l'élu se limite à la connaissance de l'électeur au moment où il lui confie ledit mandat. Dans ce sens, comment expliquer que le Parlement, qui avait été élu, en disposant du droit d'initier un référendum, en vertu de l'article 110 al. $3^{97}$ de la Constitution de 2002, ne dispose plus de cette compétence dans la Constitution de 2015, pourquoi? Bien que dans « l'ordre constitutionnel, l'interprétation [soit] extrêmement sensible au contexte politique $",{ }^{98}$ néanmoins, le continuum institutionnel soulève une série de difficultés que le seul contexte ne peut justifier. Dessaisir la représentation du peuple d'une compétence aussi importante s'apparente, pour citer Amine Ben Abdallah, à « un blocage pour le développement de la démocratie ». ${ }^{99}$

Finalement, le continuum institutionnel, contestable sur le fond, comme nous venons de le démontrer, l'est aussi quant à sa portée. En effet, ce dernier ne permet pas au pouvoir d'assurer la garantie d'une stabilisation du régime après le changement de Constitution, bien au contraire. Nous savons, avec Duguit, que « le pouvoir politique n'est jamais légitime par son origine, il peut devenir légitime par son exercice ${ }^{100}$. Dès lors, les contestations post-référendaires pouvaient se juguler grâce à une pratique politique conforme aux aspirations populaires et donc démocratiques. Ainsi, il aurait été plus convaincant, sur le plan juridique, de coupler le référendum avec une élection législative anticipée. Ou encore, coupler les élections présidentielles avec les élections législatives, pour renouveler l'ensemble du dispositif institutionnel et éviter le cafouillis institutionnel actuellement en vigueur.

En définitive, le continuum institutionnel donne l'impression que le changement constitutionnel était « au service des intérêts politiques circonstanciels. Une stratégie pour étendre hors délais constitutionnels la durée de mandats électifs, grevant ainsi d'incertitudes la

95 Article 103 « À son entrée en fonction, le Premier ministre présente devant l'Assemblée nationale le programme d'action du Gouvernement. La présentation du programme d'action du Gouvernement ne donne lieu ni à débat, ni à vote ».

96 Sur le sujet, voir, DAUGERON (V. B.), La notion d'élection en droit constitutionnel, Dalloz, 2011.

97 Art. 110 al. 3 de la Constitution de 2002 : «Il (le Parlement) a l'initiative des référendums, concurremment avec le Président de la République ».

98 ROUVILLOIS (F.), Droit constitutionnel, op.cit., p.137.

99 BEN ABDAlLAH (M. A.), « Le Parlement bicaméral aujourd'hui », Contribution à la doctrine du droit constitutionnel marocain, Rabat, REMALD, 2005, p. 136.

100 Cité par E. VILLEY, « La souveraineté nationale », RDP, 1904, p. 20. 
démocratie et l'alternance ». ${ }^{101}$ Ce sentiment se confirme aisément à la lecture de certaines dispositions de la Constitution de 2015.

\section{B. La neutralisation des verrous constitutionnels à un troisième mandat}

En droit constitutionnel, la procédure d'élaboration de la Constitution est celui du modèle démocratique classique : « le peuple est appelé à exercer le pouvoir constituant par l'élection, présentée partout comme libre et sans contrainte, d'assemblées ou de conventions chargées de rédiger et d'adopter un instrument de gouvernement $\gg .{ }^{102}$ Avec l'adoption de la Constitution de 2015, la République du Congo s'est écartée de cette procédure habituelle, ${ }^{103}$ aucun débat sur le texte en discussion n'avait été organisé. Pire, le projet de Constitution n'avait été rendu public que quelques jours avant le référendum. Au final, quelques juristes, nommés d'en haut et non choisis par le corps électoral, devaient décider du pacte social de tout un peuple.

Cette entorse au " principe de la légalité démocratique ${ }^{104}$ se justifiait pour les auteurs du changement de la Constitution, par la nécessité d'installer la " Nouvelle République ». Cette justification somme toute contestable de la « restructuration du système politique et de l'ordre constitutionnel $»^{105}$ ne peut véritablement résister à l'analyse du texte ainsi adopté.

Le postulat avancé par les tenants du changement de la Constitution était que la Constitution de janvier 2002 avait atteint son objectif - le rétablissement de la paix - comme si la paix pouvait se rétablir grâce à un texte, fut - il constitutionnel - il fallait donc changer de texte, pour arrimer le pays dans la modernité. Un tel argument impliquait une certaine audace constitutionnelle, caractérisée par une série de dispositions pouvant faire dire aux observateurs que le Congo tournait définitivement la page du présidentialisme paternaliste et

101 Pour une vision globale sur le sujet, voir, KANTE (B.), « Les juridictions constitutionnelles et la régulation des systèmes politiques en Afrique ", in Mélanges en l'honneur de Jean Gicquel, Constitutions et pouvoirs, Paris, Montchrestien, 2008, pp. 265-276.

102 LOEWENSTEIN (K.), « Réflexion sur la valeur des Constitutions dans une époque révolutionnaire. Esquisse d'une ontologie des Constitutions », in : Revue française de science politique, $2^{\mathrm{e}}$ année, ${ }^{\circ} 1,1952$, p. 6 .

103 Il est vrai que pour ce qui était de la Constitution du 20 janvier 2002, aucune assemblée constituante n'avait été élue. Toutefois, une procédure assez originale de débat constitutionnel décentralisé avait été organisée. De la sorte, les populations avaient participé au débat avant de se prononcer par référendum.

104 Ibid., p. 7.

105 VERGOTTINI (G.), Las transiciones constitucionales, Desarrollo y crisis del constitucionalismo a finales del siglo XX, Colombia, Universidad de Colombia, 2002, p.175. Traduit par Jôao Paulo Pessoa. 
autoritaire. ${ }^{106}$ Or, à l'observation de la nouvelle Constitution, l'audace n'est pas au rendezvous. Pire, le président de la République demeure le maitre incontestable du jeu politique. ${ }^{107}$ Le modernisme constitutionnel n'était donc pas le principal motif du changement de Constitution. Ceci est d'autant plus vrai qu'il aurait été possible de moderniser les institutions sans passer par la case changement. ${ }^{108}$ Ainsi, à la lecture de la Constitution de novembre 2015 , ce qui est le plus marquant, ce n'est pas " la révolution constitutionnelle », ${ }^{109}$ comme le soulignait le professeur Le Divellec, concernant l'Angleterre. ${ }^{110}$ Au contraire, c'est la disparition de tous les obstacles constitutionnels à un troisième mandat du président sortant qui marque les esprits.

Il ne s'agit pas ici de faire le récit de l'histoire constitutionnelle récente de la République du Congo. Cependant, pour bien comprendre les raisons du changement de Constitution, il faut faire un retour dans le texte constitutionnel précèdent. En effet, dans la Constitution de 2002, le pouvoir exécutif était confié au président de la République, ${ }^{111}$ élu au suffrage universel direct, son mandat était renouvelable une fois (art. 57). Par ailleurs, nul ne pouvait être élu à la présidence de la République s'il n'était âgé de moins de soixante-dix ans (art.58). Enfin, le nombre de mandats présidentiels ne pouvait faire l'objet d'une révision de la Constitution (art. 185). Ces trois articles expliquent sans doute le changement de Constitution, car ils empêchaient le président Sassou de compétir pour un nouveau mandat.

Cependant, il faut noter que les trois articles n'avaient pas la même force juridique. En effet, la limite d'âge de l'article 58 pouvait aisément faire l'objet d'une révision. En revanche, le verrou de l'article 185 semblait infranchissable, au moyen de la révision, ce qui peut expliquer ce changement assez précipité de Constitution.

Pour convaincre que la perspective d'un troisième mandat était bien au cœur du changement de Constitution, il suffit de lire l'article 245 des dispositions transitoires, qui dispo-

106 Sur la notion de présidentialisme paternaliste, nous nous permettons de renvoyer à notre article: « Le néoprésidentialisme africain: entre paternalisme et superposition ", Revue française de droit constitutionnel 2015/3 ( $\mathrm{N}^{\circ}$ 103), p. e26-e53. DOI 10.3917/rfdc.103.0792.

107 La lecture des articles 64 et 83 illustre bien notre propos : art. 64 : « Le Président de la République est le Chef de l'État. Il est garant de l'indépendance nationale, de l'intégrité du territoire et de l'unité nationale, du respect de la Constitution, des traités et accords internationaux. Il détermine la politique étrangère et de défense de la Nation. Le président de la République est garant du fonctionnement régulier des pouvoirs publics et de la continuité de l'État». Art. 83 : «Le Président de la République nomme le Premier ministre et met fin à ses fonctions. Sur proposition du Premier ministre, il nomme les autres membres du gouvernement et met fin à leurs fonctions. Le président de la République fixe par décret les attributions des membres du gouvernement ».

108 Le Titre XVIII de la Constitution de 2002, portant sur la révision était assez libéral, de sorte qu'il aurait été possible de réviser profondément sans changer, à l'image de la révision de constitutionnelle en France en 2008.

109 À propos de la loi adoptée le 15 septembre 2011, le Fixed Term Parliamentary.

110 LE DIVELLEC (A.), « Un tournant de la culture constitutionnelle britannique », Jus politicum, n ${ }^{\circ} 7,2012$.

111 Article 56 de la Constitution de 2002, faisait du chef de l'État le seul détenteur du pouvoir exécutif. 
se que: « Tout citoyen congolais, sans exclusive, qui remplit les conditions d'éligibilité, peut faire acte de candidature aux fonctions publiques électives prévues par la présente Constitution ». Cet article aux apparences sibyllines est en réalité un pare-feu efficace contre une contestation sur la rétroactivité de la réforme constitutionnelle de 2015. En effet, bien qu'ayant changé de Constitution, il aurait été possible d'opposer une fin de non-recevoir aux partisans du troisième mandat. Il aurait fallu arguer qu'ayant épuisé les nombres de mandats constitutionnels, même en présence d'un changement de Constitution, le président Sassou ne pouvait se présenter de nouveau sous l'empire de la nouvelle Constitution. Car une telle perspective confirmerait que le changement de la Constitution était simplement un moyen de contournement des verrous du texte fondamental de 2002.

Le changement n'ayant de valeur que pour l'avenir, un tel argument pouvait utilement être présenté aux partisans du troisième mandat. Dans ce sens, en 2012, la candidature de Maître Wade fut jugée anticonstitutionnelle par l'opposition. Elle estimait que l'ancien chef de l'État sénégalais ne pouvait se représenter, car l'exercice de ses fonctions était limité à deux mandats depuis la réforme de la Constitution de 2001. Élu en 2000, réélu en 2007 pour cinq ans, Abdoulaye Wade tentait de se représenter pour sept ans après le rétablissement du septennat en 2008, à la suite d'une nouvelle réforme constitutionnelle. ${ }^{12}$ Il faut dire que les deux situations ne sont pas identiques, car dans le cas sénégalais, il s'agissait de la même Constitution, ce qui avait sans doute justifié la décision contestable du Conseil constitutionnel sénégalais. ${ }^{113}$ Dans le cas du Congo, le changement de Constitution pouvait être considéré comme un nouveau départ constitutionnel, enclin à neutraliser le passé. Néanmoins, cet argument pouvait être utilisé pour éviter ce que l'on peut qualifier, avec $G$. Liet-Vaux, de « fraude à la Constitution ». ${ }^{114}$

Finalement, l'article 245 qui, de façon curieuse, énonce le fait que tout citoyen pouvait compétir à une élection - comme s'il existait un doute - visait à prévenir une éventuelle contestation sur le terrain de la rétroactivité constitutionnelle. Confirmant ainsi que le principal objectif du changement de Constitution était non pas l'évolution des institutions, mais un troisième mandat pour le président sortant.

112 Voir sur le sujet la position du professeur Ismaëla MADIOR FALL, « La recevabilité de la candidature de Me Wade », Hebdomadaire le Populaire, 30 janvier 2012, en ligne : http://www.senewe b.com/news/Politique/recevabilite-de-la-candidature-de-me-wade-le-pr-ismaela-madior-fall-pas-c onvaincu-par-la-decision-du-conseil-constitutionne_n_58436.html. Sur le même sujet, lire utilement l'article du professeur Albert BOURGI, « La candidature présidentielle de trop? », DAKARACTU, 23 février 2012, en ligne : http://www.dakaractu.com/Senegal-La-candidature-presi dentielle-de-trop-Albert-Bourgi_a14457.html.

113 Décision du Conseil constitutionnel sénégalais du 27 janvier 2012, validant la candidature de Maître Wade.

114 LIET-VAUX (G.), « La fraude à la Constitution: essai d'une analyse juridique des révolutions communautaires récentes: Italie, Allemagne, France », précité. 
En définitive, s'il est possible d'observer avec Jean du Bois de Gaudusson que « le pouvoir constituant est le pouvoir de faire la Constitution $»,{ }^{115}$ ce pouvoir ne doit pas être perçu comme un instrument conjoncturel, destiné à « porter atteinte à l'alternance et surtout de nature à modifier l'équilibre politique $» .{ }^{116}$

115 GAUDUSSON (J. D.), « Quel statut constitutionnel pour le chef de l'État en Afrique », in Le nouveau constitutionnalisme, Mélanges en l'honneur de Gérard Conac, Economica, 2001, pp. 329-337.

116 NAHM-TCHOUGLI, « Renouveau constitutionnel et État de droit en Afrique de l'Ouest francophone », A.O.A-HJF, n³ et 4, 2003. 